\title{
MOTION ANALYSIS OF HUMAN CADAVER KNEE-JOINTS USING ANATOMICAL COORDINATE SYSTEM
}

\author{
Gábor Katona $^{1}$, Béla M. Csizmadia ${ }^{1}$, István Bíró ${ }^{2}$, Kristóf Andrónyi ${ }^{3}$, \\ Gábor Krakovits ${ }^{3}$ \\ ${ }^{1}$ Szent Istvan University, Faculty of Mechanical Engineering \\ ${ }^{2}$ University of Szeged, Faculty of Engineering \\ ${ }^{3}$ Semmelweis University, Department of Orthopaedics \\ katona.gabor@gek.szie.hu
}

\begin{abstract}
Large standard deviation can be observed during the analysis of the kinematical functions which describe the motion of the knee-joint. Screening the previous large number of published results concerning to the knee motion it seemed that they are scattered, therefore it can be regarded as unreliably. In our experiments anatomical coordinate system recommended by VAKHUM project was used in favor to reduce standard deviation. Setting up the anatomical coordinate system in its special anatomical points are very uncertain. A protocol was settled for the orientation of the anatomical points and for the anatomical coordinate system. Experiments were performed on five cadaver knees using the new protocol. The measurements were analyzed which has proved that the method offers a better accuracy.
\end{abstract}

Keywords: knee joint; rotation; flexion; anatomical coordinate-system

\section{Introduction}

The knee-joint is one of the most complex joints of the human body. The scientific interest have been focusing on the biomechanics of the knee for a long time. A lot of studies, often opposite to each other are published on this scope. One of the biggest problems is to describe the motions, because an anatomical coordinate system is needed to be defined, but there is no generally accepted standard method.

The motions of knee joint can be described more precisely by means of anatomical coordinate system, which assists to compare the results of measurements of different research teams. The results of the research can be useful for surgeons as well to prosthesis designing.

Weber brothers ${ }^{16}$ dealt with the kinematics of walking in the XIX. century. The motions of the knee was analyzed by Braune ${ }^{3}$ with photographs taken from two directions. It was not possible to describe the difficult three dimensional motions on the contemporary level of technology, but the effect of passive endrotation was described by him. The angular interpretation method was framed by Grood \& Suntay $^{7}$ which was needed for the description of the knee motion (JCS - Joint Coordinate System). So, the possibility of uniform communication was established between engineers and doctors, but at the same time convenient recommendation was not given for the definition of axis of motion.

The fact that the knee joint has two axis of rotation was found by Hollister ${ }^{8}$. These are the axes of flexion-extension and axis of externalinternal rotation. Abductional motion was measured only occasionally, possible due to the different sizes of the two condyles. The transepicondylar axis is traditionally used for 
the determination of axis of flexion-extension, which is the junction-line between the lateral and medial epicondyles. It was found by Churchill $^{4}$ that the axis of flexion-extension was approximated properly by this axis, but for nowadays it seems to fall. It is confuted by a lot of studies $5,6,8$ that the two axes are the same. It is accentuated by other studies $1,9,10,12,13,17$ that the determination of the transepicondylar axis is very unsure. It can be just like $15^{\circ}$ difference between the axes in case of different researchers.

\section{Methods}

Experiments were carried on cadaver knees for the analysis of motion of the knee using an experimental test rig built by the research team. The measurements were analyzed using a (anatomical) coordinate system in favor of comparability. It is still very important to increase the precision of determination, because Bíro et al. ${ }^{2}$ have considered that large differences can be seen in the kinematical functions if the same measurement was analyzed in anatomical coordinate systems with minimal differences. Our measurements were determined on the basis of recommendation of VAKHUM project ${ }^{15}$. The anatomical coordinate system can be determined on the femur (Figure 1) on the basis of this recommendation, by using three typical anatomical points on it. These are the head of femur (fh), the medial (me) and lateral (le) epicondyles. The rules of the determination of the coordinate system are:

- the origin $\left(\mathrm{O}_{\mathrm{t}}\right)$ of the anatomical coordinate system is the midpoint of the junction-line between the medial (me) and lateral (le) epicondyles

- the $y_{t}$ axis of the coordinate system is the line between the origin and the fh point positive direction upwards

- the $x_{t}$ axis of the coordinate system is perpen-

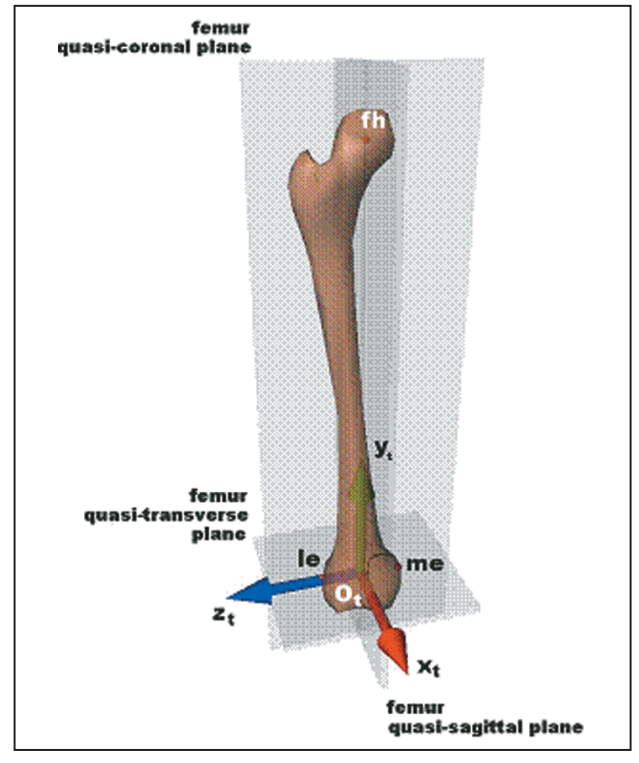

Figure 1. The anatomical coordinate system of the femur

dicular to the plane (quasi-coronal) defined by the three anatomical points (hf, me, le), positive direction to the anterior

- the $z_{t}$ axis of the coordinate system is mutually perpendicular to the $x_{t}$ and the $y_{t}$ axis with positive direction to the right.

In case of the tibia, the anatomical coordinate system can be determined by its four typical anatomical points. These anatomical points are the head of fibula (hf), tuberositas tibiae $(\mathrm{tt})$, medial $(\mathrm{mm})$ and lateral $(\mathrm{lm})$ mallelous. The rules of the determination of the coordinate system are (Figure 2$)$ :

- the origin $\left(\mathrm{O}_{\mathrm{s}}\right)$ of the coordinate system is the midpoint of the junction-line between the lateral $(\mathrm{lm})$ and medial $(\mathrm{mm})$ mallelous

- the $y_{s}$ axis of the coordinate system is the intersection of the quasi-coronal (defined by the $\mathrm{mm}, \mathrm{lm}$ hf points) and quasi-sagittal (orthogonal to the quasi-coronal plane and contains $\mathrm{O}_{\mathrm{s}}$ and tt points both) planes with positive directions upwards 


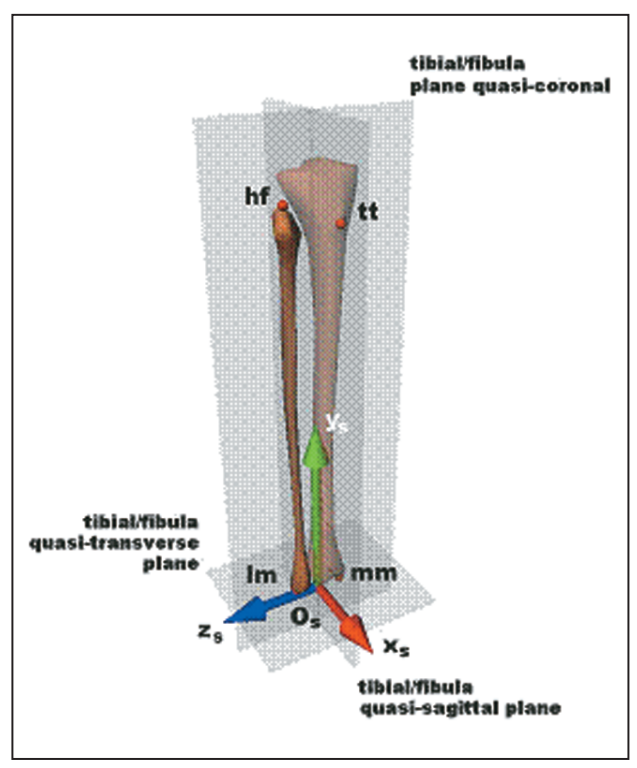

Figure 2. The anatomical coordinate system of the tibia

- the $\mathrm{x}_{\mathrm{s}}$ axis of the coordinate system is perpendicular to the quasi-coronal plane with positive direction to the anterior

- the $z_{s}$ axis of the coordinate system is mutually perpendicular to the $\mathrm{x}_{\mathrm{s}}$ and $\mathrm{y}_{\mathrm{s}}$ axis with positive direction to the right.

These anatomical points are needed to be determined during the experiments. The measurements were carried out on an experimental test rig with resected cadaver knees, so some of the anatomical points $(\mathrm{lm}, \mathrm{mm}, \mathrm{fh})$ on the whole cadaver body had to be determined. The

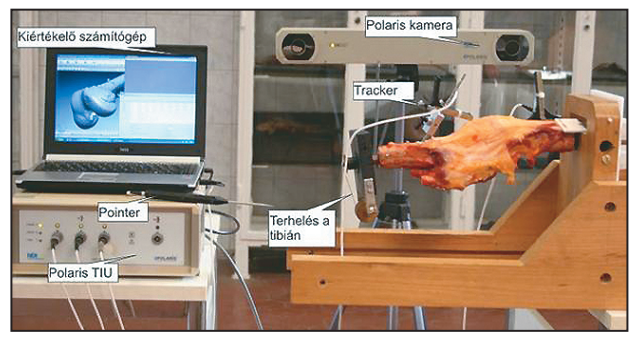

Figure 4. The experimental equipment with the Polaris system

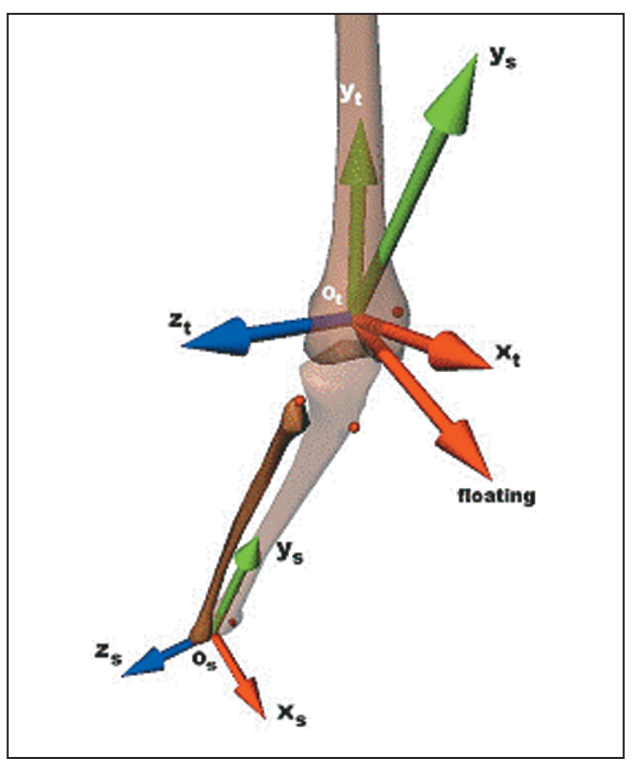

Figure 3. The joint coordinate system (JCS)

other points (me, le, hf, tt) can be determined on the whole cadaver body or on the fixed resection in the equipment also.

Polaris infrared optical positioning system (Figure 4) was used for the determination of the position of the anatomical points during the experiments ${ }^{11}$. The placed trackers' positions were measured by the Polaris system in the coordinate system fixed to the still environment. The two moving trackers were attached to the femur and the tibia so they represented rigid body. The system is suitable for measuring the location of points in the coordinate system attached to the still environment which are shown by a pointer tracker. All of the anatomical points can be marked by the pointer with exception of head of femur. The location of the head of femur can be determined only with calculation.

The head of femur can be approximated with a sphere. If an orbital motion is performed by the femur then its motion is spherical motion 
around the center of femur head. The motion of the tracker attached to the femur also a spherical motion in this case. The center of femur head can be calculated from the measurement data.

The place of the trackers of the Polaris system was changed after the necropsy of the cadaver body and fixing the knee in the equipment. The anatomical coordinate system has to be known in the equipment also for the evaluation of the measurements performed in the equipment. So every location of the anatomical points have to be measured in the absolute coordinate system of the Polaris system. But the transformation of the location of the resected anatomical points has to be ensured to the equipment.

Six marker screws (Figure 5) were used for this purpose on every bone, which were screwed in the bones. The location of them was measured on the whole body and the cadaver knee fixed in the equipment also. So the connection was established by the screws between the two states. The resected anatomical points can be transformed to the equipment by the use of the coordinate system attached to the screws.

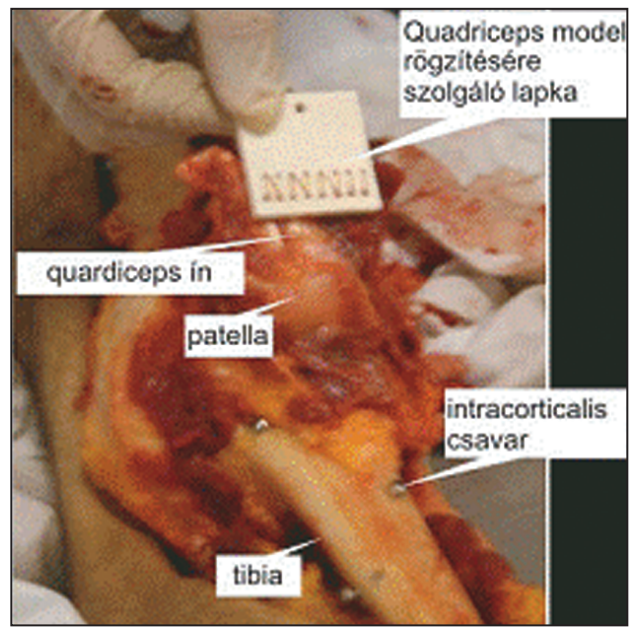

Figure 5. The prepared cadaver knee
Three marker screws are enough for the transformation, but the precision of the transformation can be increased by increasing the number of the screws.

A rubber belt which was the muscle model of the quadriceps was attached to the ligament of the quadriceps on the cadaver knee fixed in the equipment (Figure 5). The load was attached to a rod fixed in the tibia by a cord (Figure 4). The operation of the whole equipment was published by Szakál ${ }^{14}$ earlier. Two types of measurements were carried out during the experiments:

- type 1.: The load was increased which caused the flexion of the knee. The load was decreased after the total flexion of the knee till the knee went to totally stretched position.

- type 2.: The force was decreased in the rubber belt which is the muscle model of the quadriceps, so the flexion of the knee was increasing. The force was increased in the rubber belt after the total flexion till the knee went to totally stretched position.

The soft tissues were removed from the bones at the end of the measurements and the location of the anatomical points was measured in the coordinate system of the Polaris.

The use of Euler angles became general for the description of the motion of the knee. The anatomical coordinate system of the femur can be rotated into the anatomical coordinate system by these angles in case of the right order of them (Figure 3). The order of the Euler angles are: flexion-extension, abduction-adduction, external-internal rotation.

The angle of flexion-extension is the rotation around the $\mathrm{z}_{\mathrm{t}}$ axis of the anatomical coordinate system of the femur (nearly transepicondylar). The external-internal rotation is the rotation around the $y_{s}$ axis of the anatomical coordinate 
system of the tibia. The angle of abductionadduction is rotation around the axis which is mutually perpendicular to the previous two axes (floating axis).

\section{Results}

Repetitions were done from all types of the measurements at three times, because of decreasing the errors of the measurements. Six marker screws were used instead of the needed three for the transformation of the anatomical points which could also help decrease the sum of errors. The measurements can be solidly reproduced (Figure 6) and the motion of the knee is independent from the type of the measurements. The functions of rotation against flexion divides in the range of the measurements between $25-70^{\circ}$ of flexion in the range of $2^{\circ}$ of rotation. The reason of this can be that the angular speed of flexion is increased in this range, so the reception of the signals changed. The level of the rotation compared to the abduction is quite less and the most part of the changing of rotation starts in the range of starting $35^{\circ}$ of flexion.
The effect of the inaccuracy of determination of the anatomical coordinate system to the changing of functions of the kinematical parameters is detailed in the work of Bíró et al. ${ }^{2}$.

The lack of accuracy can be originated to more than one reason, for example the error of the transformation of the evaluation method. The reason of this is that the marker screws are situated on a cylinder with small diameter. Thus the angular error of the coordinate system attached to the marker screws - which requires the transformation - is relatively large. Another reason of the lack of accuracy can be also the evaluation error of the center of the head of femur. The hip of the dead body cannot be fixed totally stiffly during the measurement for the determination of the center of the head of femur. So there is a little motion of the hip during the measurement. On the other hand the measurement error of the Polaris is appeared in the results during the evaluation of the location of the head of femur.

Again the reason of the accuracy of the determination can come from the fact that the ana-

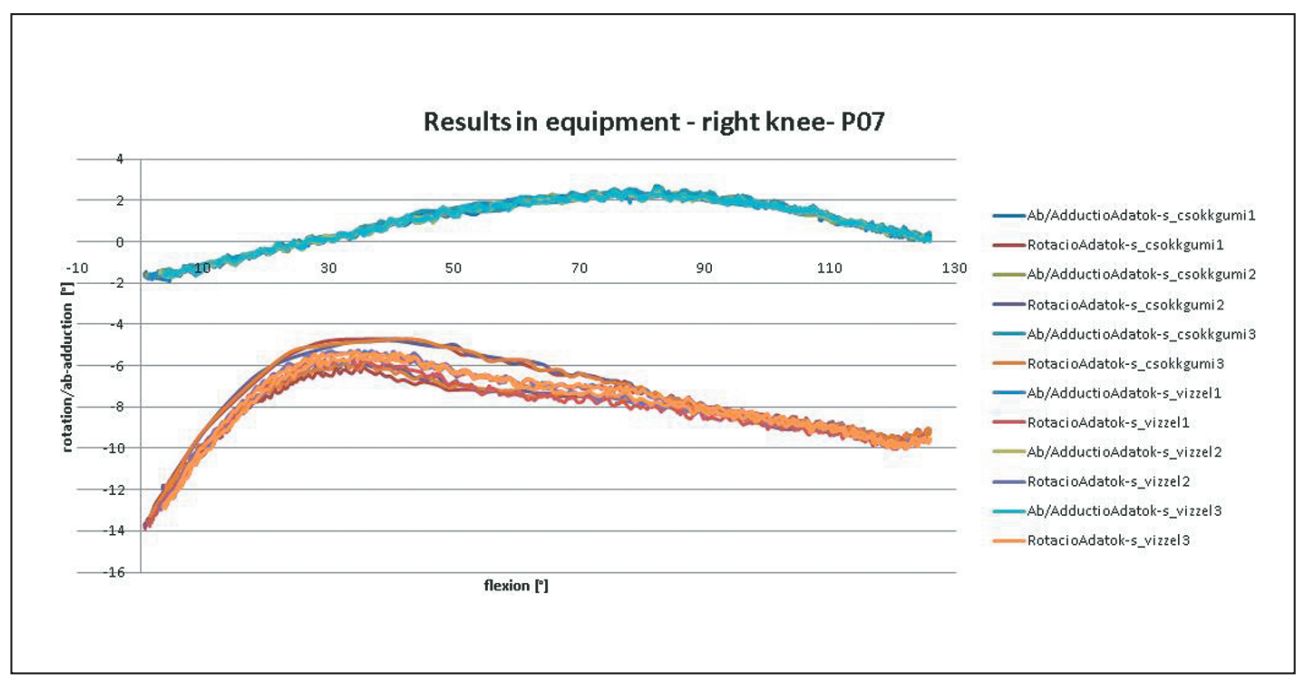

Figure 6. Rotation and ab/adduction functions against flexion in experiment no. 7 . 
tomical points are not exact, precisely definable points. They have to be marked on a finite size area.

It follows that different points are marked always at the repetition of the pointing (with the largest attention also). So, a lot of anatomical coordinate system can be determined close to each other. The anatomical points were marked in the equipment again in favor of estimation of these errors and the kinematical functions were also evaluated in the anatomical coordinate system determined this time.

The effect of the anatomical coordinate systems close to each other can be followed in the form of the functions of the same measurement (Figure 7). It can be seen that the functions of rotation against flexion are almost parallel in the range of the starting $50^{\circ}$ of the flexion. The characteristic of the functions totally changes after this range. The functions of ab/ adduction against flexion start from nearly the same value, but their characteristic are totally different.

The measurements were carried out on five cadaver knees written down previously to predict the possibility of generalization. The external-internal rotation and abductionadduction functions against flexion were represented during the evaluation of these measurements (Figure 8, Figure 9). The functions of rotation against flexion are parallel independent from different cadaver knees and the errors of anatomical coordinate systems. The functions were represented that the starting rotation and adduction were $0^{\circ}$ in favor of comparability. It can be seen, that the kinematical functions are in the same range in case of four from five knees. Deviation appeared in only one case. The reason of relatively little deviation between the four case can be the precariousness of the determination of anatomical coordinate system detailed previously. It was noticeable in case of the very different function (No.4) that the knee was in unusual valgus position which can be seen on its ab/ adduction function (Figure 9). If there is relatively large angle between the axis of flexion of the anatomical coordinate system $\left(\mathrm{z}_{\mathrm{t}}\right)$ and the transepicondylar axis then the function of external-internal rotation changes significantly (Figure 8).

It can be established that the knee starts from a lateral rotated position and ends in medial position during the flexion. The most part of

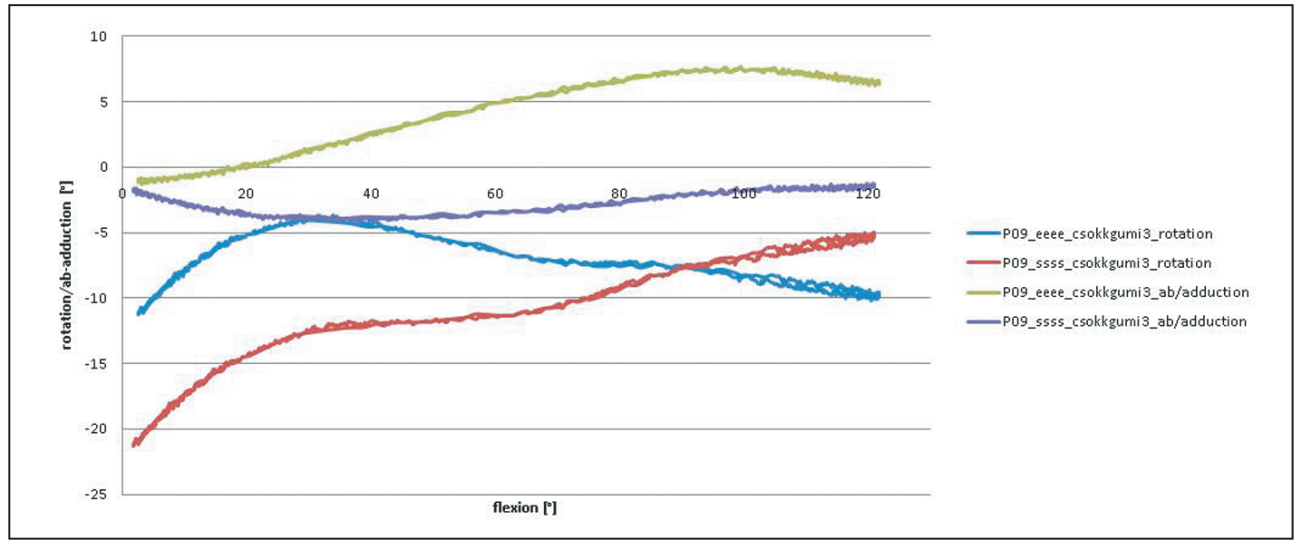

Figure 7. Rotation and ab/adduction functions against flexion of the same measurement evaluated in different anatomical coordinate system in experiment no. 9 


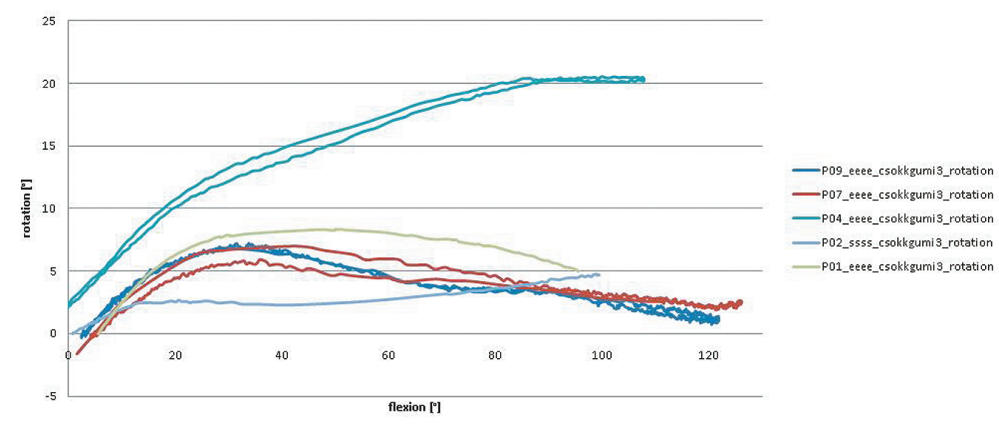

Figure 8. Rotation functions against flexion of the five experiments

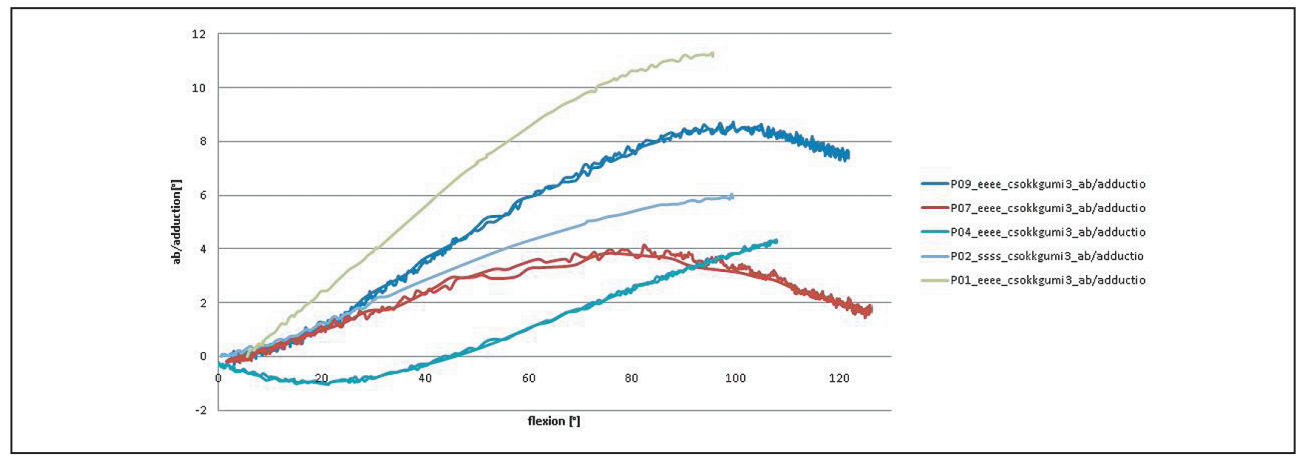

Figure 9. Ab/adduction functions against flexion of the five experiments

the changing of rotation is in the range of starting flexion around $30-35^{\circ}$. The value of the abduction-adduction is much less than the value of rotation.

\section{Conclusion}

The motion of the knee is described by the use of different anatomical coordinate systems and angles determined with different methods in the professional literature. Accordingly large standard deviation can be found in the kinematical parameters.

The following facts can be drawn on the basis of the published results that the use of our anatomical coordinate system based on the rec- ommendation of VAKHUM project is well established. The protocol is suitable for the determination of the anatomical coordinate system and for the measurement. Less standard deviation can be found in the kinematical parameters with the use of our earlier presented evaluation method.

It can be established also, that it is very important to determine the location of the anatomical points more precisely and to reduce the transformation errors during the transformation of the location of anatomical points. Experiments are performed parallel with present work for minimalization of these errors and for determination of the sensitivity to inaccuracy. 


\section{REFERENCES}

1. Aglietti $P$ et al. Rotational Position of Femoral and Tibial Components in TKA Using the Femoral Transepicondylar Axis. Clinical Orthopaedics and Related Research, 2008;466(11):2751-5.

2. Bíró I, M. Csizmadia B, and Katona G. Sensitivity investigation of three-cylinder model of human knee joint, IV. Hungarian Conference on Biomechanics, 2010.

3. Braune $W$. and Fischer O. Die Bewegungen des Kniegelenkes Nach Einer Neuen Methode am Lebenden Menschen Gemessen. 1891.

4. Churchill DL et al. The transepicondylar axis approximates the optimal flexion axis of the knee. Clinical Orthopaedics and Related Research, 1998;356:111-8.

5. Eckhoff D et al. Difference between the epicondylar and cylindrical axis of the knee. Clinical Orthopaedics and Related Research, 2007;461:238-44.

6. Eckhoff DG et al. Three-dimensional mechanics, kinematics, and morphology of the knee viewed in virtual reality. Journal of Bone \& Joint Surgery - American Volume, 2005;87(Suppl 2):71.

7. Grood ES and Suntay WJ. A joint coordinate system for the clinical description of 3-dimensional motions - application to the knee. Journal of Biomechanical Engineering-Transactions of the Asme, 1983;105(2):136-44.

8. Hollister AM et al., The axes of rotation of the knee. Clinical Orthopaedics and Related Research, 1993;290:259.

9. Jenny JY and Boeri C. Low reproducibility of the intra-operative measurement of the tran- sepicondylar axis during total knee replacement. Acta Orthopaedica Scandinavica, 2004;75(1): $74-77$.

10. Jerosch $J$ et al. Interindividual reproducibility in perioperative rotational alignment of femoral components in knee prosthetic surgery using the transepicondylar axis. Knee Surgery Sports Traumatology Arthroscopy, 2002;10(3):194-7.

11. NDI, http://www.ndigital.com/medical/ polarisfamily-techspecs.php

12. Robinson $M$ et al. Variability of landmark identification in total knee arthroplasty. Clinical Orthopaedics and Related Research, 2006;442: 57-62.

13. Stoeckl B, et al. Reliability of the transepicondylar axis as an anatomical landmark in total knee arthroplasty. Journal of Arthroplasty, 2006;21 (6):878-82.

14. Szakál Z. Méróberendezés térdízület mozgásvizsgálatához, GÉP 2006;LVII(1):37-40.

15. VAKHUM, http://www.ulb.ac.be/project/ vakhum

16. Weber WWF. Mechanik der menschlichen Gehwerkzeuge. Göttingen, 1836.

17. Yau WP et al. Errors in the identification of the transepicondylar and anteroposterior axes of the distal femur in total knee replacement using minimally-invasive and conventional approaches - A cadaver study. Journal of Bone and Joint Surgery - British Volume, 2008;90B (4):520-6.

This study was carried out with the permission of the Hungarian Scientific and Research Ethical Committee (TUKEB 165-1/2002, renewed in 2007). We would like to acknowledge the help of Gábor Renner, György Szántó, and László Szobonya from the Hungarian Academy of Sciences, Computer and Automation Research Institute.

\section{Gábor Katona}

Szent Istvan University, Faculty of Mechanical Engineering H-2100 Gödöllő, Páter Károly utca 1. 